\section{BAS/ THE MITOCHONDRIAL PERMEABILITY TRANSITION PORE BSCR45 AS A TARGET FOR CARDIOPROTECTION IN VENTRICULAR CARDIOMYOCYTES HARVESTED FROM PATIENTS WITH OBSTRUCTIVE HYPERTROPHIC CARDIOMYOPATHY}

\author{
doi:10.1136/hrt.2010.205781.56
}

${ }^{1} \mathrm{P}$ S C Rees, ' S M Davidson, ${ }^{2}$ S E Harding, ${ }^{3}$ P M Elliot, 'D M Yellon, 'D J Hausenloy. ${ }^{1}$ The Hatter Cardiovascular Institute, University College London, UK; ${ }^{2}$ The National Heart and Lung Institute, Imperial College London, UK; ${ }^{3}$ The Heart Hospital, University College London Hospitals, London, UK

Rationale The opening of the mitochondrial permeability transition pore $(\mathrm{mPTP})$ is a critical determinant of ischaemia-reperfusion injury, and preventing its opening confers powerful cardioprotection in non-diseased myocardium. Whether this cardioprotective effect is present in the setting of hypertrophic cardiomyopathy (HCM) is unknown and is investigated in this study.

Methodology Local UCLH/UCL ethical committee approval had been granted for this study. Human adult ventricular cardiomyocytes were isolated from left ventricular septal tissue, harvested from consenting patients undergoing surgical myectomy for obstructive HCM. The cells were loaded with the fluorescent dye TMRM which localises to the mitochondria, generates oxidative stress within the mitochondria on confocal imaging, resulting in mPTP opening, as indicated by the collapse of the mitochondrial membrane potential. The time taken to induce the loss of mitochondrial membrane potential was used as a measure of mPTP opening sensitivity. Cells were randomised to the following: (1) DMSO $0.01 \%$ vehicle control ( $\mathrm{N}=8 /$ group); (2) cells pre-treated with ciclosporin-A (CsA) $(0.2 \mu \mathrm{M})$ for $15 \mathrm{~min}(\mathrm{~N}=7 /$ group); (3) cells pre-treated with atorvastatin $(25 \mu \mathrm{M})(\mathrm{N}=7 /$ group $)$.

Results In the control group, mPTP opening was induced after $188.7 \% \pm 22.7 \mathrm{~s}$ of oxidative stress, providing evidence for a functional mPTP in the setting of HCM. Furthermore, pretreatment with the known mPTP inhibitor, $\mathrm{CsA}$, and atorvastatin, delayed the onset of mPTP opening by $51 \% \pm 10 \%(\mathrm{p}<0.001)$ and $35 \% \pm 7 \%$ $(p<0.05)$, respectively.

Conclusions For the first time in diseased human ventricular myocytes, we have demonstrated that the MPTP is functional and that its opening can be inhibited by cardioprotective agents such as CsA and atorvastatin.

\section{BAS/ X-BOX BINDING PROTEIN 1 SPLICING TRIGGERS AN BSCR46 AUTOPHAGIC SURVIVAL PATHWAY IN ENDOTHELIAL CELLS}

doi:10.1136/hrt.2010.205781.57

Andriana Margariti, Hongling Li, Daniel Martin, Anna Zampetaki, Yanhua Hu, Oingbo Xu, Lingfang Zeng. Cardiovascular Division, King's College London BHF Centre, London, UK

An initial step in the development of atherosclerosis is endothelial cell dysfunction. Our previous study has shown that sustained activation of X-box binding protein 1 (XBP1) splicing, a key signal transducer in endoplasmic reticulum stress response, leads to endothelial apoptosis and atherosclerosis development (Zeng et al, $P N A S$ 2009). Autophagy is characterised as a survival response as well as a pathway culminating in cell death. In this study, we aimed to investigate whether XBP1 splicing could also activate autophagy in endothelial cells, and its role in the cell survival and apoptosis. XBP1 splicing was introduced to human umbilical vein endothelial cells by adenoviral gene transfer, and autophagic vesicle formation was observed 48 and $72 \mathrm{~h}$ postinfection. Autophagy gene expression such as Beclin 1, LC3- $\beta$, NIX, ATGF-tv1, BNIP2, BNIP3, ATG5 and ATG12 was induced by XBP1 splicing as detected at RNA and protein levels. Endothelial cells transfected with the LC3 $\beta$-GFP construct showed a high GFP expression of autophagic vesicle formation only in the presence of spliced XBP1 compared with the non-infected cells. Moreover, our results showed that endostatin, a well-characterised endogenous inhibitor of angiogenesis that induces apoptosis, activated autophagy genes $4 \mathrm{~h}$ after treatment, through XBP1 splicing in an IRE1- $\alpha$-dependent manner. Knockdown of XBP1 or IRE1 $\alpha$ by ShRNA in endothelial cells ablated endostatin-induced autophagy responsive genes expression and enhanced the apoptotic effect of endostatin. These results suggest that activation of XBP1 splicing can trigger an autophagic survival signal pathway, providing novel insights into the mechanisms involved in endothelial survival.

\section{BAS/ QUANTITATIVE METABOLIC PROFILING OF SUBCLINICAL BSCR47 ATHEROSCLEROSIS BY SERUM NMR METABONOMICS}

doi:10.1136/hrt.2010.205781.58

${ }^{1,2,3} \mathrm{P}$ Würtz, ${ }^{1} \mathrm{P}$ Soininen, ${ }^{1} \mathrm{~A} \mathrm{~J}$ Kangas, ${ }^{2} \mathrm{C} \mathrm{G}$ Magnussen, ${ }^{2} \mathrm{~J} \mathrm{H}$ Raiko, ${ }^{4} \mathrm{~V}$ P Mäkinen, ${ }^{4} \mathrm{P}$ H Groop, ${ }^{4} \mathrm{R}$ Thomson, ${ }^{1} \mathrm{M} J$ Savolainen, ${ }^{2} \mathrm{M}$ Juonala, ${ }^{2} \mathrm{~J}$ Viikari, ${ }^{2} \mathrm{M}$ Kähänen, ${ }^{2} \mathrm{~T}$ Lehtimäki, ${ }^{2} \mathrm{O}$ T Raitakari, ${ }^{1} \mathrm{M}$ Ala-Korpela. ${ }^{1}$ Computational Medicine, University of Oulu, Finland; ${ }^{2}$ Cardiovascular Risk in Young Finns study group, Turku \& Tampere University Hospitals, Finland; ${ }^{3}$ Epidemiology and Public Health, Imperial College, UK; ${ }^{4}$ Folkhälsan Research Center, Finland

Objective To determine associations of systemic metabolites with subclinical atherosclerosis.

Methods 4407 serum samples were measured by nuclear magnetic resonance (NMR) spectroscopy from the Cardiovascular Risk in Young Finns Study, and carotid intima-media thickness (IMT) was assessed by ultrasound. Numerous lipoprotein lipids subclasses as well as low-molecular-weight metabolites were quantified from the NMR data.

Results In these young adults (aged $24-45$ years) data-driven analysis using self-organising maps showed distinct metabolic phenotypes associated with elevated IMT. The phenotypes were characterised by varying combinations of metabolic disturbances including elevated very-low-density lipoprotin (VLDL) and LDL subclasses, but also several low-molecular-weight metabolites. Results for 6-year incidence of high carotid IMT and IMT progression as shown by discrimination and reclassification will also be discussed. Conclusion The study showed different metabolic phenotypes inherently associated with subclinical atherosclerosis. Prediction of subclinical atherosclerosis was improved by comprehensive metabolic profiling. The findings substantiate developments towards the use of multi-metabolic risk phenotypes in cardiovascular risk assessment.

\section{BAS/ COMPUTER MOLECULAR MODELLING OF THE P22PHOX BSCR48 PROTEIN STRUCTURAL CHANGES LINKED TO C242T POLYMORPHISM}

doi:10.1136/hrt.2010.205781.59

Daniel Meijles, Brendan J Howlin, Jian-Mei Li. Cardiovascular Research, Faculty of Health and Medical Sciences, University of Surrey, UK

The p22phox is a key component of the cytochrome b558 of the NADPH oxidase (Nox), which by generating reactive oxygen species (ROS) is involved in the pathogenesis of cardiovascular disease. A p22phox polymorphism (C242T) has been found to reduce oxidative stress in the cardiovascular system and is negatively associated with the incidence of coronary heart disease (CHD). However, the mechanism involved remains unknown. In this study we used computer molecular modelling and bioinformatics to investigate the potential effect of C242T polymorphism on the 3-D protein structure of the p22phox. Based on the published sequence data of p22phox and the principle of regulated prediction algorithms, we found that p22phox consists of two domains: an N-terminal transmembrane domain (124 a.a.) and a C-terminal cytoplasmic domain (71 a.a.). In its most stable form, it has three 